\title{
Labyrinthectomy and Vestibular Neurectomy for Intractable Vertiginous Symptoms
}

\author{
Alfredo Vega Alarcón ${ }^{1,2}$ Lourdes Olivia Vales Hidalgo ${ }^{2}$ Rodrigo Jácome Arévalo ${ }^{2}$ Marite Palma Diaz ${ }^{1,2}$ \\ ${ }^{1}$ Neurology Center, Centro Médico American British Cowdray, \\ México, DF, Mexico \\ 2 Otorhinolaryngology and Neurotology Department, Instituto \\ Address for correspondence Alfredo Vega Alarcón, MD, Insurgentes \\ Sur 3877, Tlalpan. México, DF, Mexico \\ (e-mail: vega.alarcon@icloud.com).
} Nacional de Neurología y Neurocirugía Dr. Manuel Velasco Suárez, México, DF, Mexico

Int Arch Otorhinolaryngol 2017;21:184-190.

\begin{abstract}
Introduction Labyrinthectomy and vestibular neurectomy are considered the surgical procedures with the highest possibility of controlling medically untreatable incapacitating vertigo. Ironically, after 100 years of the introduction of both transmastoid labyrinthectomy and vestibular neurectomy, the choice of which procedure to use rests primarily on the evaluation of the hearing and of the surgical morbidity.

Objective To review surgical labyrinthectomy and vestibular neurectomy for the treatment of incapacitating vestibular disorders.

Data Sources PubMed, MD consult and Ovid-SP databases.

Data Synthesis In this review we describe and compare surgical labyrinthectomy and vestibular neurectomy. A contrast between surgical and chemical labyrinthectomy is also examined. Proper candidate selection, success in vertigo control and complication

\section{Keywords}

- vestibular diseases/ surgery

- labyrinth diseases/ surgery

- Ménière's disease/ surgery

- neuritis/surgery

- vertigo/surgery

- vestibular nerve/ surgery

- vestibulocochlear nerve diseases/ surgery

rates are discussed on the basis of a literature review.

Conclusions Vestibular nerve section and labyrinthectomy achieve high and comparable rates of vertigo control. Even though vestibular neurectomy is considered a hearing sparing surgery, since it is an intradural procedure, it carries a greater risk of complications than transmastoid labyrinthectomy. Furthermore, since many patients whose hearing is preserved with vestibular nerve section may ultimately lose that hearing, the long-term value of hearing preservation is not well established. Although the combination of both procedures, in the form of a translabyrinthine vestibular nerve section, is the most certain way to ablate vestibular function for patients with no useful hearing and disabling vertigo, some advocate for transmastoid labyrinthectomy alone, considering that avoiding opening the subarachnoid space minimizes the possible intracranial complications. Chemical labyrinthectomy may be considered a safer alternative, but the risks of hearing loss when hearing preservation is desired are also high.
\end{abstract}

\section{Introduction}

Robert K. Jackler and David Whinney, from the University of California in San Francisco, published in 2001 a paper entitled "A Century of Eighth Nerve Surgery", which was a scholarly review of over 70 original papers regarding the origins of surgery for vestibular disorders. ${ }^{1}$ In this work, the authors proposed a timeline that they divided in three different eras: a) The Deadly Era (1898-1930); b) The Evolutionary Era (1931-1959); and c) The Microsurgical Era received

December 2, 2016

accepted

February 1, 2017
DOI http://dx.doi.org/

$10.1055 / \mathrm{s}-0037-1599242$. ISSN 1809-9777.
Copyright (C) 2017 by Thieme-Revinter

Publicações Ltda, Rio de Janeiro, Brazil
License terms

()(1) $\Theta \circledast$ 
(from 1960 on). They described the first attempts to perform a vestibular nerve section (VNS) by early surgeons such as R.H. Parry, from the Victoria Infirmary in Glasgow, Cuthbert Wallace, from St. Thomas' London, and the contributions of Walter Dandy, from Johns Hopkins Hospital, in Baltimore, who performed 607 VNS procedures with a success rate of $90.4 \%$ for the cure of vertigo. They then moved to the introduction of labyrinthectomy techniques by Lake and Milligan in 1904, who stated that they had every reason to believe that if the intracranial division of the auditory nerve had been successful, then the direct destruction of the posterior half of the membranous labyrinth would be equally effective, with the advantage of avoiding any risk to life. The authors then continued describing variations to the latter technique, which included the injection of alcohol through the oval window or lateral semicircular canal and electrical desiccation, which were gradually abandoned because of facial nerve injury. Endolymphatic sac surgery was posteriorly proposed by Georges Portmann, from Bordeaux, in 1927, to relieve the vertigo caused by Ménière's disease. The authors stated that although this procedure might not achieve a cure rate as high as the eighth nerve section, it remains a more conservative procedure than labyrinthectomy and eighth nerve surgery, offering good results. Its consequent popularity resulted in a reduction in the number of eighth nerve sections performed over the next years. It is interesting to observe how the introduction of each therapy affects the popularity of the others. However, the last decade has witnessed a renaissance of vestibular ablation using intratympanic aminoglycosides. This procedure was introduced by Harold F. Schuknecht, from the Massachusetts Eye and Ear Infirmary in 1957.

In this review we describe and compare surgical labyrinthectomy and vestibular neurectomy. A contrast between surgical and chemical labyrinthectomy is also examined. Proper candidate selection, success in vertigo control and complication rates are discussed.

\section{Review of the Literature and Discussion}

\section{Vestibular Neurectomy}

Vestibular Neurectomy as a means of controlling incapacitating vertigo was reintroduced to otologists in 1961 by Dr. William House, by using a middle fossa approach for the management of disabling peripheral vestibulopathies. In 1980 , Silverstein and Norrell ${ }^{2}$ described vestibular neurectomy via a retrolabyrinthine approach. Since then, further refinements have been made in the technique, and now many otologists approach the vestibular division of the eight nerve via a retrosigmoid or a retrolabyrinthine craniectomy.

The retrolabyrinthine vestibular neurectomy has proven to be an excellent procedure with minimal complications ( $10 \%$ of cerebrospinal fluid leak and $3 \%$ of wound infection) according to Silverstein et al. $^{3}$ In the ideal procedure, the vestibular nerve can be transected in the cerebellopontine angle or in the internal auditory canal, depending on the presence or absence of a distinct cochleovestibular cleavage plane. The dura can be closed completely, thus avoiding a cerebrospinal fluid leak, and hearing may be unaffected.
During the 1990s, VNS became a popular method to cure the symptoms of inner ear originated vertigo while preserving hearing. In an effort to determine the results of vestibular neurectomy in the United States, a questionnaire was prepared and sent to the 350 members of the American Otologic Society and the American Neurotology Society. The results of the survey indicated that 2,820 vestibular neurectomy procedures had been performed by 58 surgeons, and that $92 \%$ of these were performed by posterior fossa approach, while only $8 \%$ were performed by middle fossa approach. $^{4}$

Traditionally, the indications for vestibular neurectomy included not only disabling Ménière's disease, in which control rates with hearing preservation approached $90 \%$, but also chronic or uncompensated vestibular neuronitis, vestibular hydrops, positional vertigo, traumatic labyrinthitis and post-stapedectomy vertigo. However, patients with diagnoses different than Ménière's did poorly with vestibular neurectomy. Of special concern were the patients with uncompensated vestibulopathy, for they had few treatment options before 1989. Since then, physical therapist-directed vestibular habituation training has been used in these patients.

Although it is generally admitted that endolymphatic sac surgery may influence both the vestibular and auditory symptoms of Ménière's disease, a positive effect of vestibular neurectomy on hearing is still the subject of controversy. Quaranta et $\mathrm{al}^{5}$ performed a comparison study of the longterm hearing results obtained after vestibular neurectomy versus endolymphatic mastoid shunt and medical therapy. In both the neurectomy and endolymphatic sac surgery groups, there were singular patients who experienced hearing improvement, but these findings seemed to be more the effect of a natural fluctuation than a benefit from the surgical procedure. Operated patients who had poor hearing initially deteriorated less than those with good hearing, and the same finding was observed in the patients who declined surgery.

Schlegel et $\mathrm{al}^{6}$ have demonstrated $95.5 \%$ of hearing function preservation, and on the vestibular side, the medium to long-term evolution of the operated patients showed that durable suppression or significant improvement of the vertigo was achieved in $90.9 \%$ of them. According to the literature, ablative surgical deafferentation, such as vestibular neurectomy, remains the most efficient procedure to treat drop attacks.

The recurrence of vertigo and dizziness in a few patients might be explained by the fact that the vestibular nerve has not been completely sectioned, leaving a residual vestibular peripheral function. Incomplete central compensation of the peripheral deafferentation may also be another cause of the remaining symptoms.

Halmagyi et $\mathrm{al}^{7}$ have shown that Ménière's disease patients with vertigo attacks and preserved hearing after successful VNS have residual semicircular canal function, predominantly in the ipsilesional posterior semicircular canal. The only vestibular nerve section patient without vertigo from their group had no residual semicircular canal function on the operated side. Head impulse, caloric, 
click-evoked vestibular myogenic potential and audiometric tests gave no evidence of the involvement of the contralesional labyrinth and cochlea, without fully excluding the possibility that some of the postoperative Ménière's attacks were the initial signs of the involvement of the contralesional ear. These results, however, suggest that residual vestibular function on the operated side, that is, an incomplete vestibular nerve section, causes postoperative vertigo attacks in Ménière's disease patients.

Anatomical and surgical data have shown that vestibular nerve topography varies from the nerve's origin in the fundus of the internal auditory canal to its entry point at the brainstem. The superior and inferior vestibular nerves merge to form the vestibular nerve. The superior part innervates the lateral and the anterior semicircular canals, and the utricle and the anterosuperior portion of the saccule, whereas the inferior part innervates the posterior semicircular canal and the remaining portion of the saccule. The precise merger point of the vestibular nerves and the cochlear nerve is subject to interindividual variations, thus making it difficult to identify their cleavage plane at operation. The proximity of the inferior vestibular and the cochlear nerves allows some vestibular fibers to occasionally cross over to the cochlear nerve, which may be spared during the vestibular nerve section. Although all patients who reported postoperative vertigo had residual posterior semicircular canal function, vestibular evoked myogenic potentials (VEMPs) reveal that about 50\% of Ménière's disease patients lose saccular function. Standard caloric tests measure only lateral semicircular canal function, and they would not have detected the residual posterior semicircular canal function responsible for the patients' persistent vertigo. These results emphasize the importance of the head impulse test to assess post-vestibular nerve section function.

Severe postoperative complications, such as facial nerve palsy and cerebrospinal fluid leakage, are reported. Therefore, the question that arises is whether it is ethically acceptable to perform an intracranial operation with potentially dangerous consequences for an inner ear condition such as Ménière's disease, which is not life-threatening. However, there is a low percentage of complications associated with damage to the facial nerve in a retrosigmoid approach, in contrast to the transtemporal vestibular nerve section. This surgical technique is also more efficient than "drainage" operations, such as endolymphatic sac decompression and shunting, sacculotomy and cochleosacculotomy, which lead to a long-term suppression of the vertigo in approximately $70 \%$ of the cases.

\section{Labyrinthectomy}

Labyrinthectomy is an extremely effective treatment for disabling episodic vertigo in patients with unilateral Ménière's disease and with other causes of intractable vertigo that have failed conservative management or hearing-sparing surgery, and in patients who have non-serviceable hearing in the affected ear. ${ }^{8}$ There are two surgical approaches to achieve labyrinthectomy: the transcanal and the transmastoid approaches. In the transmastoid labyrinthectomy, the semicircular canals and vestibule are opened and exonerated of neuroepithelium under direct visualization. In the transcanal labyrinthectomy, the vestibule is opened through a tympanotomy by removing the stapes and drilling the bone between the round and oval windows. The neuroepithelium is removed with a hook and suction. Both approaches have a high success rate in relieving patients from severe vertigo attacks.

Labyrinthectomy is effective in unstable unilateral peripheral vestibular disorders because it achieves an acute deafferentation in the pathologic ear, presuming that the patient can compensate centrally. The success rate is of $90.5 \%$ or better, and long-term outcomes are comparable to those of vestibular neurectomy. ${ }^{9}$

The use of transmastoid labyrinthectomy has been traditionally reserved for patients with poor hearing, whose vestibular symptoms are due to refractory Ménière's disease, ipsilateral delayed endolymphatic hydrops, and labyrinthitis. Typically, these patients are not only severely debilitated by their vertigo: their degree of hearing loss has progressed to such an extent that their hearing is considered nonserviceable. The precise definition of non-serviceable hearing loss has varied among practitioners, but typically encompasses either hearing classes $C$ and $D$, as defined by the American Academy of Otolaryngology-Head and Neck Surgery Committee on Hearing and Equilibrium (AAO-HNS CHE) in 1995 , or, less frequently, hearing stage 4 , as defined specifically for Ménière's disease in separate guidelines by the AAO-HNS CHE. ${ }^{10}$ Rarely, patients will have vestibular symptoms of such severity that, although they may still have usable hearing, they request to proceed with the labyrinthectomy, deliberately choosing postoperative anacusis, for the best chance of vertigo control. Conversely, patients who have serviceable hearing may complain of hearing distortion or hyperacusis, which can be so significantly debilitating that they may undergo labyrinthectomy to electively destroy all hearing function. This underlies a central facet of Ménière's disease: its subjective manifestations can be so incapacitating that those who suffer from it experience significant deterioration not only in their physical wellbeing, but in their mental and social well-beings as well. ${ }^{11}$

An area of apprehension is in the small but definite probability that a patient with a unilateral disease will develop a contralateral disease after the labyrinthectomy has already been performed. The threat of latent contralateral disease has discouraged some practitioners from implementing fully ablative procedures in the treatment of Ménière's disease. Estimates of bilateral affection from Ménière's range from $2 \%$ to $78 \%$; however, the accepted prevalence is more likely in the range of $15 \%$ to $40 \%$. There is similar variability in the literature regarding the timing of contralateral presentation in latent bilateral diseases. Some authors argue that the probability of bilateral manifestation increases with time, whereas others have observed that the risk of bilateral involvement decreases with time, and is much less likely to occur if it does not manifest within the first 5 years of symptoms. For those patients who do develop the bilateral disease, hearing in the contralateral ear typically 
remains better than in the presenting ear, with hearing reversal occurring in approximately $10 \%$ of cases.

Ablative procedures such as labyrinthectomy usually abolish vertigo, but their success in improving the quality of life depends on an adequate central vestibular compensation. Impaired compensation after surgery can result in persistent postural instability, which is exacerbated by walking or moving. Reports of incidence of disequilibrium after labyrinthectomy have ranged from 20 to $78 \%$; meanwhile, after vestibular neurectomy, the incidence of occasional unsteadiness has been shown to be between 14 and $20 \%{ }^{12}$

\section{Vestibular Neurectomy versus Labyrinthectomy}

Surgery is often performed to control the symptoms of peripheral vestibular disorders refractory to medical treatment. Two of the most reliable and commonly used methods of surgical vestibular deafferentation are transmastoid labyrinthectomy and VNS. ${ }^{13}$ Unilateral ablation of the diseased vestibular afferent provides relief from the spontaneous vertigo attacks, but central vestibular compensation is required to eliminate the severe vertigo that results from the asymmetry of the vestibular inputs created between the two ears. Incomplete vestibular compensation may also result in disability in the form of chronic disequilibrium and/or motion-provoked vertigo. The various surgical approaches to labyrinthectomy and VNS have different features that could affect vestibular compensation.

There are at least four specific differences between transmastoid labyrinthectomy and VNS that could theoretically lead to differences in postoperative vestibular compensation:

1. Histologic and physiologic studies have demonstrated that when deafferentation occurs peripheral to the vestibular ganglion (preganglionic deafferentation), as it occurs in labyrinthectomy, ganglion cells can survive, and there may be regeneration (or preservation) of spontaneous activity in the vestibular ganglion and nerve. It is unknown whether this activity fluctuates or remains stable in the long term. It is uncertain whether such neural activity would positively or negatively affect clinical vestibular compensation. Some authors have proposed that the maintenance of stable, spontaneous neural activity could accelerate the vestibular compensation process, whereas other authors have argued that such activity could lead to the failure of the procedure to control the vertigo. The few animal studies available suggest that although early static compensation is not affected by the mode of deafferentation, dynamic compensation is temporarily delayed when Scarpa's ganglion is resected.

2. When deafferentation is properly performed via labyrinthectomy, all the sensory neuroepithelium in the vestibular labyrinth can be reliably extirpated under direct visualization. On the other hand, since the anatomic division between the vestibular and auditory fibers in the eighth cranial nerve is variable, complete deafferentation of the vestibular neuroepithelium cannot always be accomplished by neurectomy in the cerebellopontine angle.
This limitation, mainly encountered in retrolabyrinthine VNS, has led some authors to favor a retrosigmoid craniotomy to allow an intrameatal exposure of the eighth nerve by the removal of the posterior lip of the internal auditory canal in the event that no well-defined septum between the auditory and vestibular fibers is identified in the cisternal portion of the eighth nerve. An incomplete sectioning of the vestibular fibers via the retrolabyrinthine approach may be a cause of surgical failure, with persistent severe attacks of vertigo. Nevertheless, there are patients with suspected or confirmed incomplete deafferentation via retrolabyrinthine VNS who are completely cured of their vertigo. The impact that retained vestibular fibers may have on vestibular compensation is unknown. It could be theorized that a "subthreshold" fluctuating signals arising in the periphery might not be strong enough to produce the subjective sensation of vertigo, but could be sufficient to impair vestibular compensation. On the other hand, a partially intact, normal peripheral input has been shown to facilitate vestibular compensation.

3. Retrolabyrinthine VNS carries greater risks of complications, and may be associated with longer postoperative recovery periods. Because laboratory evidence suggests that a "critical period" for vestibular compensation exists, during which sensory deprivation can lead to permanent detrimental effects on recovery, delayed mobilization after deafferentation could impair vestibular compensation.

4. Retrolabyrinthine VNS is an intradural procedure, and although cerebellar retraction is minimal, it can be hypothesized that nonspecific changes derived from the intradural craniotomy could affect the postoperative vestibular compensation.

Ironically, after 100 years of the introduction of both transmastoid labyrinthectomy and VNS, the choice of which procedure to use for the relief of vertigo rests primarily on the evaluation of the hearing and of the surgical morbidity. Furthermore, since many patients whose hearing is preserved with VNS ultimately lose that hearing because of ongoing peripheral pathologic changes, the long-term value of hearing preservation is not well established, rendering informed decision-making difficult. The clinical studies that have compared transmastoid labyrinthectomy and VNS have looked primarily at their efficacy in controlling the severe spells of episodic vertigo, with no data on long-term balance outcomes and vestibular compensation with objective and validated measures.

Eisenman et $\mathrm{al}^{13}$ demonstrated no significant long-term differences in physiologic compensation, self-assessment of balance function, or posturography performance between the patients successfully undergoing transmastoid labyrinthectomy or retrolabyrinth VNS to treat the peripheral vestibular pathology. There was a surprisingly high incidence of incomplete physiologic compensation in both groups, which was previously unrecognized in the clinical studies of surgery for vertigo. After a mean follow-up of more than 6 years 
postoperatively, there were also no statistically significant differences between the groups in their perceived handicaps.

These results suggest that labyrinthectomy and VNS may be recommended for the control of intractable vertigo, with no anticipated differences in the long-term recovery of balance function if the selected procedure succeeds in eliminating the attacks of spontaneous vertigo. Clinicians should understand that a significant number of patients will have evidence of incomplete vestibular compensation after either one of the procedures, but this will not necessarily correlate with the perceived balance handicap. Although very few patients will experience severe perceived balance dysfunction at long-term follow-up, at least 30\% to 50\% may have mild but clinically relevant impairments. Furthermore, the incremental risks of intracranial surgery intrinsic to vestibular neurectomy procedures must be weighed against the potential for unsatisfactory long-term hearing outcomes.

Among more than 3,500 patients who underwent surgery for the treatment of vertigo at the House Ear Institute from 1974 to 2004 , transmastoid labyrinthectomy comprised $2.7 \%$ of the procedures, while VNS comprised $20.4 \%$ (combining the translabyrinthine, retrolabyrinthine, retrosigmoid and middle cranial fossa approaches). ${ }^{14}$ Although translabyrinthine VNS is the most certain way to ablate vestibular function and has been reported to be the procedure of choice for patients with no useful hearing and disabling vertigo, some advocate for transmastoid labyrinthectomy without nerve section instead. The thought behind this choice is that avoiding opening the subarachnoid space minimizes intracranial complications.

Some of the possible reasons for failure in VNS procedures are: incomplete nerve section; nerve regeneration; concurrent disease in the central nervous system or opposite ear; new vestibular disease in the opposite ear; obstructive anatomy; the possibility of non-otologic vertigo; and other unknown causes. In addition, a few patients with apparently complete surgical deafferentation remain disabled by constant unsteadiness, possibly due to the failure of proper central nervous system compensation. Elderly patients may have more imbalance or unsteadiness problems in general, and are known to have an increased incidence of postoperative disequilibrium.

It is also important to mention that after labyrinthectomy some surviving neurons from Scarpa's ganglion may regenerate the peripheral process, which may later grow as fibrous tissue in the vestibule and form a true traumatic neuroma.

The dizziness handicap after an ablative procedure is influenced by the type of surgical procedure selected (labyrinthectomy or VNS), and by the patient's age and postoperative vestibular scores. Preoperative vestibular scores are highly associated with the emotional, physical and functional domains of the perceived handicap and the quality of life after the surgery.

\section{Labyrinthectomy versus Intratympanic Aminoglycosides}

Transtympanic perfusion of the middle ear was introduced in 1956 by Schuknecht, who used aminoglycosides for the treatment of Ménière's disease. Since then, perfusion of the inner ear with various medications became a common form of treatment for various inner ear disorders. The popularity of placing medications directly into the inner ear is attributed largely to several key advantages: the diseased ear is treated directly, without affecting the entire body; a higher concentration of medication in the inner ear can be obtained; and the systemic side effects of the drug are prevented. ${ }^{15}$

The primary route of entry of aminoglycosides into the inner ear is through the round window membrane. Alternative routes include the annular ligament of the oval window and the inner ear vasculature and lymphatic vessels. After passing through the round window membrane, solute concentration increases in the perilymphatic and endolymphatic spaces. The concentrations of the medications achieved in the inner ear fluids after perfusion are much higher than those concentrations achieved via systemic administration, particularly with steroids.

Although Schuknecht was the first to describe the transtympanic use of aminoglycosides in the treatment of Ménière's disease, interest in the procedure waned until Beck and Schmidt ${ }^{16}$ published their results on intratympanic gentamicin treatment in 1978. They reported the successful control of vertigo in more than $90 \%$ of the patients, and preservation of hearing in $42 \%$ of the patients.

Intratympanic gentamicin therapy continues to be the most prominent first-line therapy for patients with intractable vertigo related to unilateral Ménière's disease unresponsive to initial medical therapy. The goal of chemical ablation of the vestibular system in these cases is to reduce the frequency and severity of the vertigo attacks while minimizing cochlear toxicity.

Ototoxicity, manifested as hearing loss, occurs in approximately $30 \%$ of the patients treated with intratympanic gentamicin therapy. The hearing loss occurs within the first month after treatment in $80 \%$ of the patients, and it is said that a patient with hearing loss in that time frame has a $24.1 \%$ chance of recovering some hearing. The risk of hearing loss is greater when pretreatment hearing is better than 40 decibels $(\mathrm{dB})$ in pure-tone average thresholds.

Some of the main advantages of chemical perfusion of the inner ear by any transtympanic method include that it is a safe and inexpensive procedure, easily performed by any otology surgeon. Most delivery methods are minimally invasive, and can be performed in the office. With inner ear perfusion, high concentrations of medication in the inner ear can be achieved with few systemic side effects. The rates of control of the vertigo symptomatology in Ménière's disease are excellent, and that has allowed intratympanic gentamicin therapy to become the most prominent firstline treatment for intractable vertigo.

Some studies have revealed a correlation between vertigo control and vestibular ablation, also demonstrating a variety of changes in caloric responses after intratympanic gentamicin treatments. Moreover, it has been hypothesized that intratympanic gentamicin may even induce the recovery of the vestibular function by damaging the secretory functions of the vestibular dark cells, thus inhibiting or reducing endolymphatic hydrops. Horii et $\mathrm{al}^{17}$ suggested that intratympanic gentamicin injections damage vestibular hair cells 
and thereby block the neurotransmission between hair cells and the vestibular nerve, resulting in a decrease in vertigo spells. Damage to the cochlear function is the major concern of intratympanic gentamicin perfusion. A premedication audiometry is unable to predict the hearing outcome of the intratympanic gentamicin treatment.

Approximately 70 to $95 \%$ of patients with Ménière's disease are able to gain symptomatic control of their vertigo through the conservative medical management. ${ }^{18}$ For the remaining patients, more invasive procedures that produce partial or total ablation of the peripheral vestibular functions are often warranted. The initial treatments consisted of the ablation of the hearing and balance system by means of labyrinthectomy. Procedures that involved sac decompression or shunting were subsequently developed, whereas other groups were attempting to selectively damage or destroy vestibular function in the involved ear without damaging the hearing.

Labyrinthectomy is highly effective in controlling vertigo, but results in complete loss of hearing in the operative ear. The most widely used treatments for disabling Ménière's disease are vestibular neurectomy and intratympanic gentamicin injection. Both therapeutic options have good vertigo control rates, ranging from $72 \%$ to $95 \%$ for gentamicin, and from 85 to $100 \%$ for vestibular neurectomy. However, these procedures may have particular shortcomings. Intratympanic gentamicin may be complicated by sensorineural hearing loss in percentages ranging from zero to $95 \%$ because of the intrinsic ototoxic nature of this drug. The dose of gentamicin to be injected, the frequency of the injections, the number of doses, and the clinical end point of the therapy are still not well understood. Post-treatment hearing deterioration has also been reported for VNS, with a 27 to $50 \%$ incidence of hearing decrease defined as a shift of $10 \mathrm{~dB}$ in pure-tone average or a decrease of $15 \%$ on the speech discrimination score testing. Total deafness is also described as a rare event. As mentioned earlier, VNS also shows potential risks associated with the surgery, including intraoperative bleeding, postoperative cerebrospinal fluid leak and meningitis.

Coletti et $\mathrm{al}^{18}$, from the Verona group, have reported that vestibular nerve section has a higher rate of vertigo control with less risk of damaging hearing than intratympanic gentamicin, and, therefore, they consider it the treatment of choice for disabling Ménière's disease. Their experience with gentamicin has been quite discouraging for both hearing and disability, since they have observed a high prevalence of hearing deterioration ( $20.8 \%$ of marked deterioration) and lower levels in a functionality scale.

Still, intratympanic gentamicin maintains an important role in the treatment of vertigo associated with Ménière's disease in a particular category of subjects. In fact, although hearing is likely to be at greater risk with intratympanic gentamicin than with vestibular nerve section, there are many situations with concomitant medical problems or apprehension about undergoing surgical intervention. In addition, when the pretreatment hearing in the affected ear is very poor and hearing rehabilitation is impossible, further damage to the remaining hearing may be inconsequential.

Several studies have reported the hearing loss rate and vertigo control with vestibular nerve section and gentamicin treatment separately. Schlegel et $\mathrm{al}^{6}$ have demonstrated that the results after VNS proved to be more consistent than those reported after chemical deafferentation of the labyrinth with aminoglycosides. The outcome after chemical labyrinthectomy using aminoglycosides is often satisfactory, but unpleasant ataxia, more intense tinnitus, and a postoperative decrease in hearing are not rare. Chemical labyrinthectomy can achieve good results with regards to the suppression of vertigo and drop attacks, but VNS has the advantage of preserving the hearing function better. Data has also shown that in some patients, the hearing function even improved after retrosigmoid VNS. The underlying mechanism of this improvement remains unexplained.

\section{Final Comments}

Both VNS and labyrinthectomy may achieve a high and comparable rate of vertigo control. Even though vestibular neurectomy is considered a hearing sparing surgery, since it is an intradural procedure, it carries a greater risk of complications than transmastoid labyrinthectomy. Furthermore, since many patients whose hearing is preserved with VNS ultimately lose that hearing because of ongoing peripheral pathologic changes, the long-term value of hearing preservation is difficult to establish. Although the combination of both procedures, such as a translabyrinthine VNS, is the most certain way to ablate vestibular function and, for that, it is considered the procedure of choice for patients with no useful hearing and disabling vertigo, some still advocate for transmastoid labyrinthectomy without nerve section, considering that the intracranial complications are minimized by avoiding opening the subarachnoid space. Chemical labyrinthectomy may be seen as a safer option, but the risks of hearing loss when hearing preservation is desired are high, and vestibular ablation is not as consistent as that obtained with the aforementioned surgical alternatives.

Conflict of Interest

The authors declare no conflicts of interest.

\section{References}

1 Jackler RK, Whinney D. A century of eighth nerve surgery. Otol Neurotol 2001;22(03):401-416

2 Molony TB. Decision making in vestibular neurectomy. Am J Otol 1996;17(03):421-424

3 Silverstein H, Norrell H, Smouha E, Jones R. Combined retrolabretrosigmoid vestibular neurectomy. An evolution in approach. Am J Otol 1989;10(03):166-169

4 Silverstein H, Wanamaker H, Flanzer J, Rosenberg S. Vestibular neurectomy in the United States-1990. Am J Otol 1992;13(01): 23-30

5 Quaranta A, Onofri M, Sallustio V, Iurato S. Comparison of longterm hearing results after vestibular neurectomy, endolymphatic 
190 Labyrinthectomy and Vestibular Neurectomy for Intractable Vertiginous Symptoms Alarcón et al.

mastoid shunt, and medical therapy. Am J Otol 1997;18(04): 444-448

6 Schlegel M, Vibert D, Ott SR, Häusler R, Caversaccio MD. Functional results and quality of life after retrosigmoid vestibular neurectomy in patients with Ménière's disease. Otol Neurotol 2012;33(08):1380-1385

7 Lehnen N, Aw ST, Todd MJ, Halmagyi GM. Head impulse test reveals residual semicircular canal function after vestibular neurectomy. Neurology 2004;62(12):2294-2296

8 Sargent EW, Liao E, Gonda RL Jr. Cochlear patency after transmastoid labyrinthectomy for Ménière's syndrome. Otol Neurotol 2016;37(07):937-939

9 Heidenreich KD, Griffin GR, Lorenz MB, Telian SA. Transmastoid labyrinthectomy for disabling vertigo in a patient with internal auditory canal pathology. Otol Neurotol 2011;32(01):122-124

10 Committee on Hearing and Equilibrium guidelines for the diagnosis and evaluation of therapy in Menière's disease. American Academy of Otolaryngology-Head and Neck Foundation, Inc. Otolaryngol Head Neck Surg 1995;113(03):181-185

11 Diaz RC, LaRouere MJ, Bojrab DI, Zappia JJ, Sargent EW, Shaia WT. Quality-of-life assessment of Ménière's disease patients after surgical labyrinthectomy. Otol Neurotol 2007;28(01):74-86
12 Badke MB, Pyle GM, Shea T, Miedaner J. Outcomes in vestibular ablative procedures. Otol Neurotol 2002;23(04):504-509

13 Eisenman DJ, Speers R, Telian SA. Labyrinthectomy versus vestibular neurectomy: long-term physiologic and clinical outcomes. Otol Neurotol 2001;22(04):539-548

14 De La Cruz A, Borne Teufert K, Berliner KI. Transmastoid labyrinthectomy versus translabyrinthine vestibular nerve section: does cutting the vestibular nerve make a difference in outcome? Otol Neurotol 2007;28(06):801-808

15 Hoffmann KK, Silverstein H. Inner ear perfusion: indications and applications. Curr Opin Otolaryngol Head Neck Surg 2003;11(05): 334-339

16 Beck C, Schmidt CL. 10 years of experience with intratympanally applied streptomycin (gentamycin) in the therapy of Morbus Menière. Arch Otorhinolaryngol 1978;221(02):149-152

17 Horii A, Saika T, Uno A, et al. Factors relating to the vertigo control and hearing changes following intratympanic gentamicin for intractable Ménière's disease. Otol Neurotol 2006;27(06): 896-900

18 Colletti V, Carner M, Colletti L. Auditory results after vestibular nerve section and intratympanic gentamicin for Ménière's disease. Otol Neurotol 2007;28(02):145-151 